\title{
SOME AXIALLY SYMMETRIC POTENTIAL PROBLEMS
}

\author{
by F. G. LEPPINGTON and H. LEVINE
}

(Received 23rd June 1971)

\begin{abstract}
Some axially symmetric boundary value problems of potential theory are formulated as integral equations of the first kind. In each case the kernel admits an expansion, for small values of a parameter of the problem, that leads to an approximate integral equation whose solution provides a direct asymptotic estimate for the physical quantity of primary interest. A manipulation of the original and modified integral equations provides an efficient formula for calculating higher order terms in the asymptotic expansion.
\end{abstract}

\section{Introduction}

A variety of methods is available for the analysis of boundary value problems in axisymmetric potential theory (cf. Sneddon (10)), though explicit solutions seldom result and practical objectives are met by establishing the initial terms of series expansions for individual cases. If more than one characteristic length is involved in the geometrical description of a problem, expansions for an integrated quantity such as the total charge or flux take on distinctive forms according as these lengths are comparable in magnitude or not. Expansions of an irregular type, involving powers and logarithms of a small parameter (which is related to the difference of a pair of lengths, say) are more difficult to specify and relatively few representatives from this category are known with any degree of completeness.

It is our intention in this paper to show that details of irregular or singular perturbation expansions for linear boundary value problems of potential theory are furnished without elaborate calculation if a suitable integral equation formulation is devised at the outset; a stream function is sometimes appropriate for the latter purpose and integral equations on infinite rather than finite intervals may be used to advantage.

The particular integral equations which form the basis for our analysis are all of the first kind and, despite the unavailability of exact solutions, prove to be advantageous insofar as

(1) the quantity of interest for each problem, which is related to an integral of the solution, appears explicitly and linearly therein, and 
(2) the kernels admit expansion for small values of the relevant parameter so that analytic approximation can be obtained directly or the quantity of interest estimated by comparison with a fully determinate integral equation of independent origin.

When a first approximation to the solution of a basic integral equation is known, with a concomitant evaluation for the quantity of interest, an efficient representation of the difference between this estimate and the exact value, obtained by manipulation of the original and modified integral equation, facilitates the derivation of additional or higher order terms in the sought-for expansion.

The technique is applied to several boundary value problems in what follows, corroborating or extending results obtained by Spence $(11,12)$ through intricate transform analysis for two planar configurations that involve an annular disc or gap, confirming a prediction by Kirchhoff (5) for the thickness effect in a circular disc condenser and complementing a calculation by Fock (3) which pertains to the conductivity of a circular aperture in a transverse section of a cylindrical tube.

\section{The electrified annular disc}

An annular disc of inner and outer radii $a$ and $b$ is held at unit potential, and it is required to find its capacity when $a$ and $b$ are nearly equal. If polar coordinates $(r, \theta, z)$ are chosen so that the disc occupies the region $z=0$, $a \leqq r \leqq b$, then the symmetry of the configuration clearly implies that the potential $\phi(r, z)$ is independent of $\theta$, and is an even function of $z$. Thus we may confine our attention to the half-space $z \geqq 0$, with $\phi(r, z)$ specified as the function that vanishes at infinity and satisfies the conditions

$$
\left.\begin{array}{c}
\left(\frac{\partial^{2}}{\partial r^{2}}+\frac{1}{r} \frac{\partial}{\partial r}+\frac{\partial^{2}}{\partial z^{2}}\right) \phi=0, \quad z \geqq 0 \\
\phi=1, \quad z=0, a<r<b \\
\phi_{z}=0, \quad z=0, r<a \text { or } r>b,
\end{array}\right\}
$$

where the notation $\phi_{z}$ means $\partial \phi / \partial z$.

The problem can readily be reduced to that of an integral equation of the first kind, by applying Green's theorem to the potential $\phi(r, z)$ and a Green's function $G\left(\boldsymbol{r} ; \boldsymbol{r}_{1}\right)$ chosen to have vanishing normal derivative on the plane $z=0$. Thus

where

$$
\phi\left(r_{1}, z_{1}\right)=\int_{0}^{2 \pi} d \theta \int_{a}^{b} r G\left(r, \theta, 0 ; r_{1}, \theta_{1}, z_{1}\right) \phi_{z}(r, 0) d r, \quad z_{1}>0
$$

$$
G=-\frac{1}{4 \pi R_{+}}-\frac{1}{4 \pi R_{-}} ; R_{ \pm}=\left(r^{2}+r_{1}^{2}-2 r r_{1} \cos \left(\theta-\theta_{1}\right)+\left(z \pm z_{1}\right)^{2}\right)^{\frac{1}{2}}
$$


Formula (2.2) gives the potential at any point $r_{1}$ in terms of the unknown charge density

$$
\sigma(r)=-\frac{1}{4 \pi} \phi_{z}(r, 0)
$$

on the upper surface. In particular, if $r_{1}$ lies on this surface then $\phi$ assumes its prescribed value unity, and we have the integral equation

$$
1=\int_{a}^{b} K\left(r, r_{1}\right) r \sigma(r) d r, \quad a<r_{1}<b,
$$

for the charge $\sigma$, where the kernel $K\left(r, r_{1}\right)$ is given by

$$
\begin{aligned}
K\left(r, r_{1}\right) & =2 \int_{0}^{2 \pi}\left(r^{2}+r_{1}^{2}-2 r r_{1} \cos \theta\right)^{-\frac{1}{2}} d \theta \\
& =\frac{8}{\left(r+r_{1}\right)} K\left(\frac{2 r^{\frac{1}{2}} r_{1}^{\frac{1}{2}}}{r+r_{1}}\right)
\end{aligned}
$$

in terms of the elliptic integral

$$
K(\alpha)=\int_{0}^{\pi / 2}\left(1-\alpha^{2} \sin ^{2} \theta\right)^{-\frac{1}{2}} d \theta .
$$

The capacity $C$ of the annulus is equal to the total charge divided by the potential; thus

$$
C=2 \int_{a}^{b} 2 \pi r \sigma(r) d r .
$$

Now the integral equation (2.5), which is valid for all values of the parameters $a$ and $b$, is particularly well suited to the case of interest here, with the two radii nearly equal. To exploit the fact that the pair of variables $r$ and $r_{1}$ appearing in (2.5) differ little from $a$, we define the small dimensionless parameter

$$
\varepsilon=(b / a)-1
$$

and rescale the variables by the transformation

$$
r=a(1+\varepsilon x), \quad r_{1}=a\left(1+\varepsilon x_{1}\right)
$$

so that $x$ and $x_{1}$ vary between 0 and 1. It is also convenient to normalise the charge density according to the formula

$$
V(x)=\frac{4 \pi a \varepsilon}{C} r \sigma(r)=\frac{4 \pi a \varepsilon}{C}(a+a \varepsilon x) \sigma(a+a \varepsilon x) .
$$

In terms of the function $V(x)$, the integral equation (2.5) and the expression (2.8) take the form

$$
\frac{4 \pi}{C}=\int_{0}^{1} K\left(x, x_{1}\right) V(x) d x, \quad 0<x_{1}<1,
$$


and

$$
1=\int_{0}^{1} V(x) d x
$$

where $K\left(x, x_{1}\right)$ denotes $K\left(r, r_{1}\right)$ with $r=1+\varepsilon x$ and $r_{1}=1+\varepsilon x_{1}$.

The kernel $K$, given by (2.6), can readily be expanded for small values of $\varepsilon$, using the known expansion (cf. Gradstein and Rhysik (9)) for the elliptic integral of order close to unity; an expansion of this type is carried out in a similar problem by Grinberg and Kuritsyn (4). It is found that, as $\varepsilon \rightarrow 0$,

$K\left(x, x_{1}\right) \sim-\frac{4}{a}\left\{\log \varepsilon+\log \frac{\left|x-x_{1}\right|}{8}-\frac{1}{2}\left(x+x_{1}\right) \varepsilon \log \varepsilon-\frac{1}{2} \varepsilon \log \frac{\left|x-x_{1}\right| e}{8}\right\}+o(\varepsilon)$.

A first approximation $V_{0}(x)$ for the unknown function $V(x)$, and a corresponding estimate $C_{0}$ for the capacity, is now obtained from the governing equations $(2.12,2.13)$ on replacing the exact kernel $K$ by the first pair of terms in the uniformly valid expansion (2.14). If we define

$$
K_{0}\left(x, x_{1}\right)=-\frac{4}{a}\left\{\log \varepsilon+\log \frac{\left|x-x_{1}\right|}{8}\right\}
$$

then $V_{0}(x)$ and $C_{0}$ satisfy the equation

i.e.

$$
\frac{4 \pi}{C_{0}}=\int_{0}^{1} K_{0}\left(x, x_{1}\right) V_{0}(x) d x, \quad 0<x_{1}<1,
$$

$$
\frac{a}{C_{0}}+\frac{1}{\pi} \log \frac{\varepsilon}{8}=-\frac{1}{\pi} \int_{0}^{1} V_{0}(x) \log \left|x-x_{1}\right| d x,
$$

if $V_{0}(x)$ is normalised to satisfy the scaling condition (2.13).

Such an integral equation, with a logarithmic kernel, can readily be inverted: its solution, given by several authors (cf. Cooke (2)) is

$$
V_{0}(x)=\frac{1}{\log 4}\left\{\frac{a}{C_{0}}+\frac{1}{\pi} \log \frac{\varepsilon}{8}\right\} x^{-\frac{1}{2}}(1-x)^{-\frac{1}{2}},
$$

whence integration between 0 and 1 , and use of the condition (2.13), leads to the results

and

$$
C_{0}^{-1}=(\pi a)^{-1} \log (32 / \varepsilon),
$$

$$
V_{0}(x)=\pi^{-1} x^{-\frac{1}{2}}(1-x)^{-\frac{1}{2}}
$$

An improved estimate for the function $V(x)$, and hence for the capacity $C$ can in principle be obtained from the governing equations $(2.12,2.13)$, on the basis of an iteration scheme using $V_{0}(x)$ as a first approximation. In particular, since the kernel $K$ is seen from (2.14) to differ from $K_{0}$ by a term of order $\varepsilon \log \varepsilon$, such a scheme readily provides the information that the next term in the expansion of $V(x)$ is also of the order $\varepsilon \log \varepsilon$. Higher order terms are obtained 
along similar lines in a related low frequency wave problem by Grinberg and Kuritsyn (4).

A more efficient procedure for gaining a direct improvement on our estimate (2.19) for $C$ is now shown to be possible, using only the first approximation $V_{0}(x)$ together with the exact equations (2.12), (2.16) satisfied by $V(x)$ and $V_{0}(x)$. For if (2.16) is multiplied by $V\left(x_{1}\right)$ and integrated from 0 to 1 , then

$$
\frac{4 \pi}{C_{0}}=\int_{0}^{1} \int_{0}^{1} K_{0}\left(x, x_{1}\right) V_{0}(x) V\left(x_{1}\right) d x d x_{1},
$$

on account of the scaling condition (2.13). Similarly, if (2.12) is multiplied by $V_{0}\left(x_{1}\right)$,

$$
\frac{4 \pi}{C}=\int_{0}^{1} \int_{0}^{1} K\left(x, x_{1}\right) V(x) V_{0}\left(x_{1}\right) d x d x_{1},
$$

whence, on subtraction, the symmetry of $K\left(x, x_{1}\right)$ yields the formula

$$
\frac{1}{C}-\frac{1}{C_{0}}=\frac{1}{4 \pi} \int_{0}^{1} \int_{0}^{1} V_{0}(x) V\left(x_{1}\right)\left\{K\left(x, x_{1}\right)-K_{0}\left(x, x_{1}\right)\right\} d x d x_{1} ;
$$

this is an exact expression for the difference between $C$ and its estimate $C_{0}$, given by (2.19).

A first approximation for the error (2.21) is obviously obtained by replacing $V\left(x_{1}\right)$ by $V_{0}\left(x_{1}\right)$, and using the expansion (2.14) to estimate $K-K_{0}$. Further, since $V$ differs from $V_{0}$ by a term of order $\varepsilon \log \varepsilon$, the form of the expansion (2.14) for $K\left(x, x_{1}\right)$ shows that the first two terms of the integral (2.21) are obtained by replacing $V$ by $V_{0}$ and using the two leading terms in the expansion for $K-K_{0}$. Since $C_{0}^{-1}$ already contains two terms in the expansion for the capacity, the formula $(2.21)$ is seen to be very efficient in providing the four leading terms for $C^{-1}$ in terms of only the first approximation $V_{0}(x)$ for $V$.

Thus from (2.20), and $(2.14,2.15)$, we get

$$
\begin{aligned}
& \frac{1}{C}-\frac{1}{C_{0}}=\frac{1}{2 a \pi^{3}} \int_{0}^{1} \int_{0}^{1} x^{-\frac{1}{2}}(1-x)^{-\frac{1}{2}} x_{1}^{-\frac{1}{2}}\left(1-x_{1}\right)^{-\frac{1}{2}}\left\{\left(x+x_{1}\right) \varepsilon \log \varepsilon\right. \\
& \left.\quad+\varepsilon \log \frac{\left|x-x_{1}\right| e}{8}+o(\varepsilon)\right\} d x d x_{1}=\frac{1}{2 a \pi^{3}}\left\{\pi^{2} \varepsilon \log \varepsilon+\varepsilon \pi^{2} \log (e / 32)\right\}+o(\varepsilon) .
\end{aligned}
$$

Finally, from (2.19),

$$
\frac{1}{C}=\frac{1}{2 \pi a}\left\{2 \log \frac{32}{\varepsilon}+\varepsilon \log \varepsilon+\varepsilon \log \frac{e}{32}\right\}+o(\varepsilon),
$$

which is in agreement with the result due to Spence (11), who expresses his result in non-dimensional form by multiplying by the known capacity $C^{*}=2 b / \pi=2 a(1+\varepsilon) / \pi$ for the disc of radius $b$. Thus

$$
C^{*} / C=\pi^{-2}\{-2 \log \varepsilon+2 \log 32-\varepsilon \log \varepsilon+\varepsilon \log (32 e)+o(\varepsilon)\}
$$




\section{An electrified circular disc with an infinite coplanar screen}

A complementary problem is that posed by Spence (12), in which a disc of radius $a$, held at unit potential, is separated by an annular gap of width $b-a$ from an earthed screen that extends from $r=b$ to $r=\infty$. The symmetry about the plane $z=0$ containing the plates leads to a boundary value problem for the axisymmetric potential function $\phi(r, z)$ in the half-space $z \geqq 0$. Thus

$$
\left.\begin{array}{rl}
\left(\frac{\partial^{2}}{\partial r^{2}}+\frac{1}{r} \frac{\partial}{\partial r}+\frac{\partial^{2}}{\partial z^{2}}\right) \phi=0, \quad z \geqq 0 \\
\phi=1, \quad z=0, r<a, \\
\phi_{z}=0, \quad z=0, a<r<b, \\
\phi=0, \quad z=0, r>b,
\end{array}\right\}
$$

and we are again concerned with finding the capacity of the system when the gap is small, i.e. for small values of the parameter $\varepsilon=(b / a)-1$.

Our aim is to reduce the problem (3.1) to an integral equation of the first kind, over the region $a<r<b$, so that an approximate solution may be obtained by the procedure of the previous section. Such an equation is most readily derived by introducing a suitable stream function for the problem, in the manner described in (7) and (8). This function $\psi(r, z)$ is defined by the formulae

$$
r \phi_{r}=r \psi_{z} \text { and } r \phi_{z}=-\frac{\partial}{\partial r}(r \psi)
$$

with $\psi=0$ when $r=0, z=0$, this implying that $\psi(0, z)$ is zero for all positive $z$.

The boundary value problem for $\psi(r, z)$ resulting from (3.1) and (3.2) has the specifications

$$
\left.\begin{array}{rl}
\left(\frac{\partial^{2}}{\partial r^{2}}+\frac{1}{r} \frac{\partial}{\partial r}+\frac{\partial^{2}}{\partial z^{2}}-\frac{1}{r^{2}}\right) \psi=0, \quad z \geqq 0 \\
\psi_{z}=0, \quad z=0, r<a, \\
\psi=C / r, \quad z=0, a<r<b, \\
\psi_{z}=0, \quad z=0, r>b ;
\end{array}\right\}
$$

the constant $C$ is an unknown of the problem, to be found from the scaling condition that the potential assumes the constant value unity on the inner disc, whence

$$
-1=\int_{a}^{b} \phi_{r}(r, 0) d r=\int_{a}^{b} \psi_{z}(r, 0) d r .
$$

An essential feature of this formulation is that the constant $C$ appearing in the specifications (3.3) is the numerical value of the capacity of the system. For the capacity, which is the total charge on the disc divided by the potential 
difference, is therefore equal to twice the charge on the upper surface $r<a$, $z=0$, whence

$$
\text { capacity }=-\frac{2}{4 \pi} \int_{0}^{a} \phi_{z}(r, 0) 2 \pi r d r=\int_{0}^{a} \frac{\partial}{\partial r}(r \psi) d r=C .
$$

The stream function $\psi(r, z)$ at any point $z \geqq 0$ can be represented in terms of the normal derivative $\psi_{z}(r, 0)=\phi_{r}(r, 0), a<r<b$, through the use of Green's theorem applied to $\psi(r, z)$ and a Green's function $G\left(r ; r_{1}\right)$ that has zero normal derivative across the plane $z=0$.

Thus

$$
\begin{aligned}
\psi\left(r_{1}, z_{1}\right) & =\int_{0}^{2 \pi} d \theta \int_{a}^{b} G\left(r, \theta, 0 ; r_{1}, \theta_{1}, z_{1}\right) \psi_{z}(r, 0) r d r, & & z_{1}>0 \\
& =\int_{a}^{b} g\left(r, 0 ; r_{1}, z_{1}\right) \psi_{z}(r, 0) r d r, & & z_{1}>0,
\end{aligned}
$$

where $g\left(r, z ; r_{1}, z_{1}\right)$ is specified by the conditions

$$
\left(\frac{\partial^{2}}{\partial r^{2}}+\frac{1}{r} \frac{\partial}{\partial r}+\frac{\partial^{2}}{\partial z^{2}}-\frac{1}{r^{2}}\right) g=\frac{1}{r} \delta\left(r-r_{1}\right) \delta\left(z-z_{1}\right), \quad g_{z}\left(r, 0 ; r_{1}, z_{1}\right)=0 \text {. }
$$

In particular, if $a<r_{1}<b$ and $z_{1} \rightarrow 0$, then $\psi$ is seen from (3.3) to take the value $C / r_{1}$, and the radial electric field in the gap, $\psi_{2}(r, 0)=\phi^{\prime}(r)$, satisfies the integral equation

$$
C=\int_{a}^{b} K\left(r, r_{1}\right) \phi^{\prime}(r) d r, \quad a<r_{1}<b .
$$

The symmetric kernel $K\left(r, r_{1}\right)=r r_{1} g\left(r, 0 ; r_{1}, 0\right)$ is given in (7) by the formula

$$
K\left(r, r_{1}\right)=\frac{2}{\pi} r_{>}\left\{\mathrm{E}\left(\frac{r_{<}}{r_{>}}\right)-\mathrm{K}\left(\frac{r_{<}}{r_{>}}\right)\right\},
$$

where $r_{<}$and $r_{>}$denote the lesser and greater of the variables $r$ and $r_{1} ; \mathbf{E}$ and $K$ are complete elliptic integrals, $K$ being given by (2.7) and

$$
\mathbf{E}(\alpha)=\int_{0}^{\pi / 2}\left(1-\alpha^{2} \sin ^{2} \theta\right)^{\frac{1}{2}} d \theta
$$

Equation (3.7) and the scaling condition (3.4) are of the same type as the corresponding pair $(2.12,2.13)$ discussed in section 2 , and can be dealt with by exactly the same procedure for small values of $\varepsilon=(b / a)-1$. Thus if the rescaled variables $\left(x, x_{1}\right)$ of $(2.10)$ are used in place of $\left(r, r_{1}\right)$, and if we define

$$
U(x)=-a \varepsilon \phi^{\prime}(a+a \varepsilon x)
$$

then the governing equations $(3.7,3.4)$ become

$$
-C=\int_{0}^{1} K\left(x, x_{1}\right) U(x) d x, \quad 0<x_{1}<1,
$$


with

$$
1=\int_{0}^{1} U(x) d x
$$

The kernel $K\left(x, x_{1}\right)$, which means $K\left(r, r_{1}\right)$ written in terms of $\left(x, x_{1}\right)$, can easily be expanded for small values of $\varepsilon$, making use of the known results (cf. Gradstein and Rhysik (9)) for the elliptic integrals.

It is found that

$$
K=K_{0}+\frac{a}{2 \pi}\left\{\left(x+x_{1}\right) \varepsilon \log \varepsilon+\varepsilon\left(x+x_{1}\right) \log \frac{\left|x-x_{1}\right| e}{8}+o(\varepsilon)\right\}
$$

where

$$
K_{0}=\frac{a}{\pi} \log \frac{\varepsilon e^{2}}{8}+\frac{a}{\pi} \log \left|x-x_{1}\right|
$$

Proceeding as in section 2, a first approximation $U_{0}(x)$, and $C_{0}$, is obtained by replacing $K$ by $K_{0}$ in the integral equation (3.11). Thus

and

$$
\left.\begin{array}{rl}
-C_{0} & =\int_{0}^{1} K_{0}\left(x, x_{1}\right) U_{0}(x) d x \\
1 & =\int_{0}^{1} U_{0}(x) d x,
\end{array}\right\}
$$

with the solution

and

$$
U_{0}(x)=\frac{1}{\log 4}\left\{\frac{C_{0}}{a}+\frac{1}{\pi} \log \frac{\varepsilon e^{2}}{8}\right\} x^{-\frac{1}{2}}(1-x)^{-\frac{1}{2}}=\frac{1}{\pi} x^{-\frac{1}{2}}(1-x)^{-\frac{1}{2}},
$$

$$
C_{0}=\frac{a}{\pi}\left\{-\log \varepsilon+\log \frac{32}{e^{2}}\right\}
$$

An improved estimate follows, as in section 2, from the exact formula

$$
C-C_{0}=-\int_{0}^{1} \int_{0}^{1}\left(K-K_{0}\right) U\left(x_{1}\right) U_{0}(x) d x d x_{1}
$$

whence from (3.13) and (3.16),

$$
\begin{aligned}
C-C_{0}=-\frac{a}{2 \pi^{3}} & \int_{0}^{1} \int_{0}^{1} x^{-\frac{1}{2}}(1-x)^{-\frac{1}{2}} x_{1}^{-\frac{1}{2}}\left(1-x_{1}\right)^{-\frac{1}{2}} \\
& \left\{\left(x+x_{1}\right) \varepsilon \log \varepsilon+\varepsilon\left(x+x_{1}\right) \log \frac{\left|x-x_{1}\right| e}{8}+o(\varepsilon)\right\} d x d x_{1} .
\end{aligned}
$$

Finally then, from (3.17) and (3.19), we get

$$
C=\frac{a}{\pi}\left\{-\log \varepsilon+\log \frac{32}{e^{2}}-\frac{1}{2} \varepsilon \log \varepsilon-\frac{1}{2} \varepsilon \log \frac{e}{32}+o(\varepsilon)\right\}
$$

in which the first two terms are those obtained by Spence (12). 


\section{A condenser with parallel circular dises of finite thickness}

Two identical circular discs, each of unit radius and thickness $h$, are held at equal and opposite potentials +1 and -1 , and form a condenser whose capacity $C$ is to be found when the separation $2 \varepsilon$ and thickness $h$ are small compared with the radius.

An earlier analysis (7), dealing with the case of zero thickness, $h=0$, has provided the first few terms of the asymptotic expansion for $C$, in agreement with results obtained by Kirchhoff (5) and others. The approach developed in (7), which is now extended to include finite thickness, is to exploit the fact that the potential $\phi$ near the end faces closely resembles that of the corresponding two-dimensional problem involving a pair of semi-infinite plates. An exact integral equation formulation, to determine the potential, is derived and compared with the corresponding equations for the analogous two-dimensional problem; a direct comparison of the respective equations is sufficient to determine the capacity to a high degree of accuracy.

The symmetry of the problem implies that the potential $\phi$ is zero over the plane midway between the discs, so that we may confine our attention to the half-space above this plane. A polar coordinate system $(r, \theta, z)$ is chosen so that the symmetry plane is $z=-\varepsilon$, with the disc at potential +1 occupying the region $r \leqq 1,0 \leqq z \leqq h$. The boundary value problem to determine the potential $\phi(r, z)$ is now reduced to that of a pair of coupled integral equations over the domains $z=0, r>1$ and $z=h, r>1$, again by introducing a suitable stream function for the problem.

Working in the half-space $z \geqq-\varepsilon$, it is convenient to subdivide into the three regions $z \geqq h, 0 \leqq z \leqq h$, and $-\varepsilon \leqq z \leqq 0$, these being denoted respectively by the subscripts 1,2 and 3 (Fig. 1). The stream function $\psi(r, z)$ is defined by the formulae

$$
r \phi_{r}=r \psi_{z}, \quad r \phi_{z}=-\frac{\partial}{\partial r}(r \psi)
$$

with constants of integration chosen so that

$$
\psi_{1}(0, h)=0, \quad \psi_{3}(0,0)=0, \quad \text { and } \lim _{r \rightarrow \infty} r \psi_{2}(r, z)=0 .
$$

The boundary value problem for $\psi(r, z)$ is given by

$$
\begin{gathered}
\left(\frac{\partial^{2}}{\partial r^{2}}+\frac{1}{r} \frac{\partial}{\partial r}-\frac{1}{r^{2}}+\frac{\partial^{2}}{\partial z^{2}}\right) \psi=0, \\
\psi_{z}=0, r \leqq 1, z=0 \text { or } z=h, \\
\psi_{z}=0, z=-\varepsilon, \\
\frac{\partial}{\partial r}(r \psi)=0, r=1,0 \leqq z \leqq h .
\end{gathered}
$$


The choice of constants of integration defined by (4.2) implies that $r \psi$ is discontinuous across the planes $z=h$ and $z=0$,

i.e.

$$
\psi_{1}-\psi_{2}=A / r, \quad z=h, \quad r \geqq 1,
$$

and

$$
\psi_{2}-\psi_{3}=B / r, \quad z=0, \quad r \geqq 1 \text {. }
$$

region 1

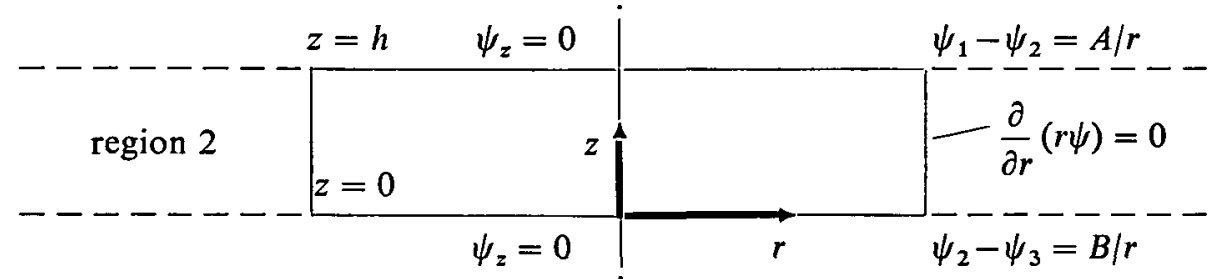

region 3

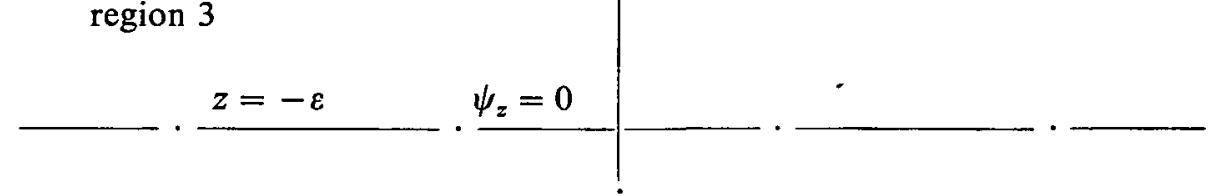

FIG. 1

The constants $A$ and $B$ are to be found, and are related to the capacity $C$ of the system. For the total charge on the upper surface is

$$
\Sigma_{1}=-\int_{0}^{1} \frac{1}{4 \pi} \frac{\partial \phi}{\partial z}(r, h) 2 \pi r d r=\frac{1}{2} \int_{0}^{1} \frac{\partial}{\partial r}\left(r \psi_{1}\right) d r=\frac{1}{2} \psi_{1}(1, h),
$$

and the charge on the lower surface is given similarly as

$$
\Sigma_{3}=-\frac{1}{2} \psi_{3}(1,0),
$$

while the curved surface, $r=1,0 \leqq z \leqq h$, carries a charge

$$
\Sigma_{2}=-2 \pi \int_{0}^{h} \frac{1}{4 \pi} \frac{\partial \phi}{\partial r}(1, z) d z=-\frac{1}{2}\left\{\psi_{2}(1, h)-\psi_{2}(1,0)\right\} .
$$

It follows that the capacity $C=\frac{1}{2}\left(\Sigma_{1}+\Sigma_{2}+\Sigma_{3}\right)$ is related to the constants $A$ and $B$ by the formula

$$
C=\frac{1}{4}(A+B) \text {. }
$$

Integral equation formulation. A representation for the stream function $\psi_{1}\left(r_{1}, z_{1}\right)$, for any point in region $1,\left(z_{1} \geqq h\right)$ is obtained by choosing a Green's function $g_{1}\left(r, z ; r_{1}, z_{1}\right)$ that vanishes at infinity and has zero normal derivative on the plane $z=h$. Specifically,

$$
\psi_{1}\left(r_{1}, z_{1}\right)=\int_{1}^{\infty} g_{1}\left(r, h ; r_{1}, z_{1}\right) r \phi_{r}(r, h) d r, \quad z_{1} \geqq h,
$$


where $g_{1}$ is such that

with details given in (7).

$$
g_{1}\left(r, h ; r_{1}, h\right)=\frac{2}{\pi r_{<}}\left\{\mathrm{E}\left(\frac{r_{\leq}}{r_{>}}\right)-\mathrm{K}\left(\frac{r_{<}}{r_{>}}\right)\right\},
$$

A similar analysis for region $3\left(-\varepsilon \leqq z_{1} \leqq 0\right)$ requires a Green's function that has vanishing normal derivative on each of the planes $z=0$ and $z=-\varepsilon$, whence

$$
\psi_{3}\left(r_{1}, z_{1}\right)=-\int_{1}^{\infty} g_{3}\left(r, 0 ; r_{1}, z_{1}\right) r \phi_{r}(r, 0) d r, \quad-\varepsilon \leqq z_{1} \leqq 0,
$$

where the Green's function,

$$
g_{3}\left(r, z ; r_{1}, z_{1}\right)=-\frac{1}{2 \varepsilon} \frac{r_{\leq}}{r_{>}}-\frac{2}{\varepsilon} \sum_{1}^{\infty} \cos \frac{n \pi z}{\varepsilon} \cos \frac{n \pi z_{1}}{\varepsilon} I_{1}\left(\frac{n \pi r_{<}}{\varepsilon}\right) K_{1}\left(\frac{n \pi r_{>}}{\varepsilon}\right) \text {, }
$$

expressed in terms of the modified Bessel functions $I_{1}$ and $K_{1}$, is the same as that appearing in the analysis (7) for discs of zero thickness.

The corresponding formula for the region 2 requires a Green's function $g_{2}\left(r, z ; r_{1}, z_{1}\right)$ specified by the conditions

$$
\left(\frac{\partial^{2}}{\partial r^{2}}+\frac{1}{r} \frac{\partial}{\partial r}-\frac{1}{r^{2}}+\frac{\partial^{2}}{\partial z^{2}}\right) g_{2}=\frac{1}{r} \delta\left(r-r_{1}\right) \delta\left(z-z_{1}\right)
$$

with $\left(g_{2}\right)_{z}=0$ when $r \geqq 1, z=0$ or $h$, and $r\left(g_{2}\right)_{r}+g_{2}=0$ when $r=1$, $0 \leqq z \leqq h$. Thus we find, for $0 \leqq z_{1} \leqq h$,

$$
\psi_{2}\left(r_{1}, z_{1}\right)=-\int_{1}^{\infty} g_{2}\left(r, h ; r_{1}, z_{1}\right) r \phi_{r}(r, h) d r+\int_{1}^{\infty} g_{2}\left(r, 0 ; r_{1}, z_{1}\right) r \phi_{r}(r, 0) d r,
$$

with $g_{2}$ given by

$$
\begin{aligned}
g_{2}=- & \frac{1}{2 h} \frac{r_{\leq}}{r_{>}}-\frac{2}{h} \sum_{1}^{\infty} \cos \frac{n \pi z}{h} \cos \frac{n \pi z_{1}}{h} I_{1}\left(\frac{n \pi r_{<}}{h}\right) K_{1}\left(\frac{n \pi r_{>}}{h}\right)+\frac{1}{2 h} \frac{r}{r_{1}} \\
& +\frac{2}{h} \sum_{1}^{\infty} \cos \frac{n \pi z}{h} \cos \frac{n \pi z_{1}}{h} \frac{I_{1}\left(\frac{n \pi}{h}\right)+\frac{n \pi}{h} I_{1}^{\prime}\left(\frac{n \pi}{h}\right)}{K_{1}\left(\frac{n \pi}{h}\right)+\frac{n \pi}{h} K_{1}^{\prime}\left(\frac{n \pi}{h}\right)} K_{1}\left(\frac{n \pi r}{h}\right) K_{1}\left(\frac{n \pi r_{1}}{h}\right) .
\end{aligned}
$$

Finally, the discontinuity conditions $(4.4,4.5)$ are applied, using the three formulae (4.7), (4.9) and (4.11), and the problem is reduced to the following pair of coupled integral equations for the radial fields $\phi_{r}(r, 0)$ and $\phi_{r}(r, h)$ :

$\int_{1}^{\infty} \phi_{r}(r, h) r r_{1}\left(g_{1}+g_{2}\right)\left(r, h ; r_{1}, h\right) d r-\int_{1}^{\infty} \phi_{r}(r, 0) r r_{1} g_{2}\left(r, 0 ; r_{1}, h\right) d r=A$

E.M.S.-E 
and

$-\int_{1}^{\infty} \phi_{r}(r, h) r r_{1} g_{2}\left(r, h ; r_{1}, 0\right) d r+\int_{1}^{\infty} \phi_{r}(r, 0) r r_{1}\left(g_{2}+g_{3}\right)\left(r, 0 ; r_{1}, 0\right) d r=B$,

for $r_{1} \geqq 1$, with the constants $A$ and $B$ determined from the requirement that $\phi=1$ on the disc, whence

$$
\int_{1}^{\infty} \phi_{r}(r, h) d r=\int_{1}^{\infty} \phi_{r}(r, 0) d r=-1
$$

Approximation near the edges. The coupled equations (4.13) and (4.14), which are, in principle, sufficient to determine the solution for any values of the parameters $\varepsilon$ and $h$, are now shown to be particularly useful for the case of interest here, when $\varepsilon$ and $h$ are small. For although their exact solution is not possible, some information regarding the desired constants $A$ and $B$ can be obtained by simply comparing (4.13) and (4.14) with the corresponding equations for a pair of semi-infinite plates of the same thickness and separation. The argument is that, for points sufficiently close to the end faces (i.e. $r_{1}$ close to unity), the potential will be insensitive to the small curvature of the discs, as was found to be the case in the earlier work (7) on the problem of discs with zero thickness.

Thus we write

$$
r=1+x, \quad r_{1}=1+x_{1},
$$

and expand the kernels of the equations (4.13) and (4.14) for small values of $x$ and $x_{1}$. This is a simple procedure since the Bessel functions $I_{1}$ and $K_{1}$, that appear in $g_{2}$ and $g_{3}$, have uniformly large arguments and may be replaced by their asymptotic forms in terms of exponentials, and the sums can readily be performed. Denoting the approximate solution so obtained by the suffix 0 , the integral equations (4.13) and (4.14) reduce to the form

$$
\begin{gathered}
\int_{0}^{\infty} \phi_{0 x}(x, h)\left\{\frac{\left(x-x_{<}\right)}{h}+\frac{1}{\pi} \log \left[\left|x-x_{1}\right|\left(1-e^{-\pi\left|x-x_{1}\right| / h}\right)\left(1-e^{-\pi\left|x+x_{1}\right| / h}\right)\right]\right\} d x \\
-\int_{0}^{\infty} \phi_{0 x}(x, 0)\left\{\frac{\left(x-x_{<}\right)}{h}+\frac{1}{\pi} \log \left[\left(1+e^{-\pi\left|x-x_{1}\right| / h}\right)\left(1+e^{-\pi\left|x+x_{1}\right| / h}\right)\right]\right\} d x \\
=A_{0}+\frac{1}{\pi} \log \frac{e^{2}}{8}, \\
-\int_{0}^{\infty} \phi_{0 x}(x, h)\left\{\frac{\left(x-x_{<}\right)}{h}+\frac{1}{\pi} \log \left[\left(1+e^{-\pi\left|x-x_{1}\right| / h}\right)\left(1+e^{-\pi\left|x+x_{1}\right| / h}\right)\right]\right\} d x \\
+\int_{0}^{\infty} \phi_{0 x}(x, 0)\left\{\frac{\left(x-x_{<}\right)}{h}+\frac{1}{\pi} \log \left[\left(1-e^{-\pi\left|x-x_{1}\right| / h}\right)\left(1-e^{-\pi\left|x+x_{1}\right| / h}\right)\right.\right. \\
\left.\left.\left(1-e^{-\pi\left|x-x_{1}\right| / \varepsilon}\right)\right]-\frac{x_{<}}{\varepsilon}\right\} d x=B_{0}-\frac{1}{2 \varepsilon},
\end{gathered}
$$


for $x_{1}>0$, where use has been made of the additional scaling conditions that

$$
\int_{0}^{\infty} \phi_{0 x}(x, h) d x=\int_{0}^{\infty} \phi_{0 x}(x, 0) d x=-1 .
$$

Although the approximate equations $(4.17,4.18)$ still look difficult to deal with, it is to be noted that they are derived only for comparison with those appropriate to the corresponding two-dimensional configuration, for which a solution can be obtained by conformal mapping. It will now be shown that the two-dimensional equations differ from $(4.17,4.18)$ only in that definite constants appear on the right-hand sides: a direct comparison is therefore sufficient to determine $A_{0}$ and $B_{0}$ without the need to solve the equations.

Two-dimensional problem. The corresponding two-dimensional problem, whose solution leads to a first approximation $A_{0}, B_{0}$ for the constants $A, B$, is that of a pair of semi-infinite plates of thickness $h$ and separation $2 \varepsilon$, maintained at potentials \pm 1 . Exploiting the symmetry about the mid-plane, we have to find a harmonic function $\Phi(x, y)$ that vanishes on the plane $y=-\varepsilon$, and takes the value unity on the three surfaces $(x \leqq 0, y=h),(x \leqq 0, y=0)$ and $(x=0,0 \leqq y \leqq h)$.

This problem is readily solved by means of the following conformal transformation from the complex plane $z=x+i y$ to $\zeta=\xi+i \eta$ :

$$
\begin{gathered}
z=\zeta^{\frac{1}{t}}(\zeta+\alpha)^{\frac{1}{2}}+\left(\beta+\frac{1}{2} \alpha\right) \cosh ^{-1}\left(\frac{2 \zeta}{\alpha}+1\right)-\beta^{\frac{1}{2}}(\alpha+\beta)^{\frac{1}{2}} \cosh ^{-1} \frac{(1+2 \beta / \alpha) \zeta+\beta}{\zeta-\beta} \\
-i \pi \beta^{ \pm}(\alpha+\beta)^{\frac{1}{2}},
\end{gathered}
$$

(cf. Kober (6)), where the constants $\alpha$ and $\beta$ are given in terms of $\varepsilon$ and $h$ by the formulae

$$
\pi \beta^{\frac{1}{2}}(\alpha+\beta)^{\frac{1}{2}}=\varepsilon, \quad \pi\left(\beta+\frac{1}{2} \alpha\right)=\varepsilon+h .
$$

The transformation (4.20) is found to map the plate $A B C D$ and the line $E F$ on to the real $\xi$-axis (Fig. 2).
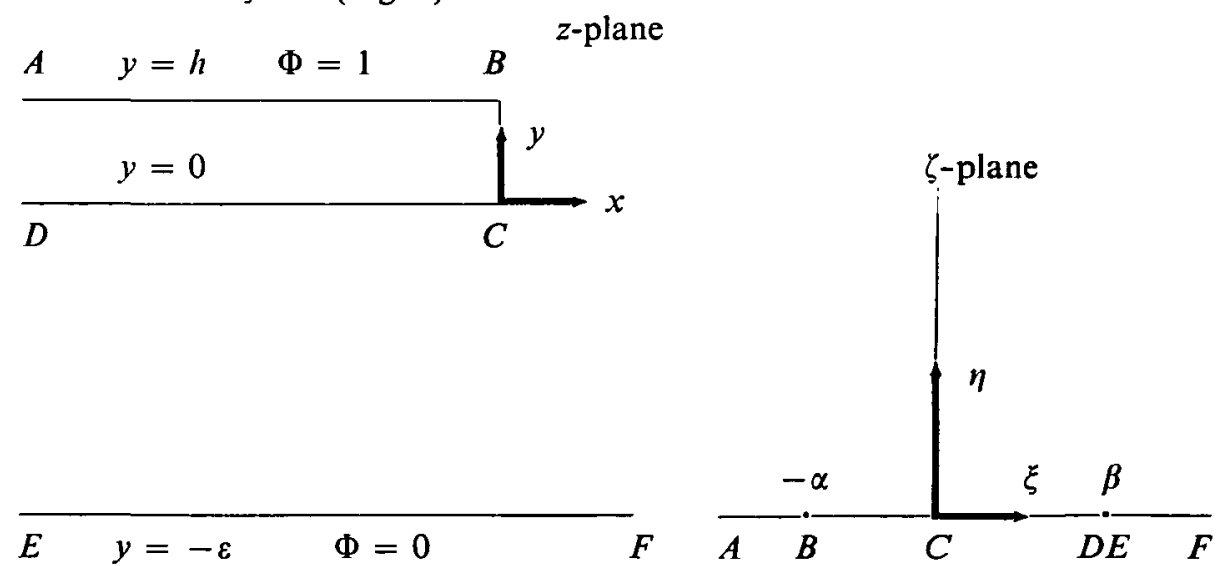

FIG. 2 
The required complex potential $\Omega=\Phi+i \Psi$ is easily calculated in terms of $\zeta$, and is found to be

$$
\Omega(\zeta)=-\frac{i}{\pi} \log \left(\frac{\zeta-\beta}{\beta}\right)
$$

An explicit solution in terms of $x$ and $y$ is not possible, since the transformation (4.20) cannot be inverted in a simple form; but the solution (4.22), together with (4.20), can be used to infer the limiting form of the solution at large distance from the origin, for points both between and outside the plates. For points between the plates, it is found that

where

$$
\Psi(x, y) \sim-x / \varepsilon+P \text { as }|z| \rightarrow \infty, \text { through } D E,
$$

$$
P=\frac{1}{\pi}\left\{1+\frac{\beta+\frac{1}{2} \alpha}{\beta^{\frac{1}{2}}(\alpha+\beta)^{\frac{1}{2}}} \cosh ^{-1}\left(\frac{2 \beta}{\alpha}+1\right)-\log \frac{4}{\alpha}(\alpha+\beta)\right\} .
$$

For points outside the plates, on the other hand,

$$
\Psi(x, y) \sim-\frac{1}{\pi} \log \left(x^{2}+y^{2}\right)^{\frac{1}{2}}+\frac{1}{\pi} \log \beta \text { as }|z| \rightarrow \infty, \text { through } A F .
$$

A pair of coupled integral equations for $\Phi_{x}(x, 0)$ and $\Phi_{x}(x, h)$, corresponding to $(4.13,4.14)$ for the discs, can readily be derived by a similar procedure. Using the asymptotics $(4.23,4.25)$ to calculate the contributions from arcs at infinity, it is found that the equations for $\Phi_{x}(x, 0)$ and $\Phi_{x}(x, h)$ are exactly like (4.17) and (4.18), with the constants on the right replaced by

$$
\begin{aligned}
& \left.(1-\log \beta) / \pi \text { instead of } A_{0}+\frac{1}{\pi} \log \frac{e^{2}}{8}\right) \\
& \text { and } \\
& P-1 / \pi \quad \text { instead of } B_{0}-1 / 2 \varepsilon
\end{aligned}
$$

Approximate evaluation of the capacity. Since the governing equations for $\phi_{0}$ and $\Phi$ differ only by the scaling constants on the right, and since each function has the same scaling condition (4.19), the pair must be identically equal. Thus the constant terms of their integral equations can be equated, and the constants $A_{0}$ and $B_{0}$ are seen from (4.26) to have the values

$$
\left.\begin{array}{l}
A_{0}=-\frac{1}{\pi} \log \frac{e^{2}}{8}+\frac{1}{\pi}-\frac{1}{\pi} \log \beta \\
\text { and } \\
B_{0}=1 / 2 \varepsilon+P-1 / \pi
\end{array}\right\} .
$$

Finally, the capacity $C=\frac{1}{4}(A+B)$ takes the approximate form

$$
\begin{aligned}
C \sim C_{0} & =\frac{1}{8 \varepsilon}-\frac{1}{4 \pi} \log \left(\frac{e^{2} \beta}{8}\right)+\frac{1}{4} P \\
& =\frac{1}{8 \varepsilon}+\frac{1}{4 \pi} \log \left\{\frac{8 \pi}{e \varepsilon}\left(1+\frac{h}{2 \varepsilon}\right)\right\}+\frac{1}{8 \pi} \frac{h}{\varepsilon} \log \left(1+\frac{2 \varepsilon}{h}\right),
\end{aligned}
$$


from (4.24), and using (4.21) to express the answer in terms of $\varepsilon$ and $h$. The result (4.28) is precisely that proposed by Kirchhoff (5).

The infinite range of integration in the exact formulae $(4.13,4.14)$ implies that the asymptotic approximation of the kernels leading to $(4.17,4.18)$ is not uniform, therefore not strictly valid. The justification for this step is that for values of $r$ significantly greater than unity, for which the asymptotics are not valid, the functions $\phi_{r}(r, h)$ and $\phi_{r}(r, 0)$ are very small on account of the dipole nature of the field away from the discs. This point is discussed more fully in the analysis (7) for discs of zero thickness, for which an improved estimate for the capacity is derived. It is likely that the next term in the expansion for $C$ could be obtained similarly here, by manipulating the exact equations $(4.13,4.14)$ and the approximate equations $(4.17,4.18)$ to provide efficient formulae for $A$ and $B$, analogous to (2.21) and (3.18) in the earlier sections.

\section{Steady flow through a constricted circular tube}

A problem investigated by Fock (3) concerns the steady flow of current down a circular tube, of radius $b$, across which is placed a plane screen containing a circular aperture, of radius $a$, whose centre is on the axis of the tube. Fock deals with the case when the aperture radius $a$ is small compared with $b$, while the present investigation concerns the complementary problem when the two radii are nearly equal.

Cylindrical polar coordinates $(r, \theta, z)$ are chosen so that the tube is given by $r=b,-\infty<z<\infty$, with the screen occupying the region $a<r<b, z=0$. The scalar potential $\phi(r, z)$ is a harmonic function whose asymptotic form $\phi \sim A z$, for large $|z|$, corresponds to the free flow down the tube with no constriction, where $A$ is a constant proportional to the total current $I$. The symmetry of the problem implies that $\phi(r, z)$ is an odd function of $z$, so that we may consider only the region $z \geqq 0$, in which $\phi$ is specified by the conditions

$$
\left.\begin{array}{rl}
\left(\frac{\partial^{2}}{\partial r^{2}}+\frac{1}{r} \frac{\partial}{\partial r}+\frac{\partial^{2}}{\partial z^{2}}\right) \phi=0, \quad z \geqq 0 \\
\phi=0, \quad z=0, r<a, \\
\phi_{z}=0, \quad z=0, a<r<b, \\
\phi_{r}=0, \quad z \geqq 0, r=b \\
\phi \sim A z+B \quad \text { as } z \rightarrow \infty,
\end{array}\right\}
$$

where $A$ is given and $B$ is an unknown constant to be found.

In order to obtain an integral equation representation over the region $z=0, a \leqq r \leqq b$, we follow the lead given in sections 3 and 4 by defining the stream function $\psi(r, z)$ :

$$
r \phi_{r}=r \psi_{z} \text { and } r \phi_{z}=-\frac{\partial}{\partial r}(r \psi)
$$

E.M.S. -E2 
with the condition that $\psi(0,0)=0$. This implies that $\psi(0, z)=0$ along the whole $z$-axis, whence on taking the limit $r \rightarrow 0$ in the second of formulae (5.2) we have

$$
\phi_{z}(0, z)=-2 \psi_{r}(0, z), \quad z \geqq 0 .
$$

The boundary value problem for $\psi(r, z)$ is found from (5.1) and (5.2) to be:

$$
\begin{aligned}
\left(\frac{\partial^{2}}{\partial r^{2}}+\frac{1}{r} \frac{\partial}{\partial r}-\frac{1}{r^{2}}+\frac{\partial^{2}}{\partial z^{2}}\right) \psi=0, \quad z \geqq 0, \\
\psi_{z}=0, \quad z=0, r<a \\
\psi=-\frac{1}{2} A b^{2} / r, \quad z=0, a<r<b \\
\psi=-\frac{1}{2} A b, \quad z \geqq 0, r=b \\
\psi \sim-\frac{1}{2} A r, \quad \text { as } z \rightarrow \infty .
\end{aligned}
$$

The total current $I$ flowing down the tube is given from conditions at large $|z|$ as

$$
I=\sigma \int_{0}^{b} \frac{\partial \phi}{\partial z} 2 \pi r d r=\sigma \pi b^{2} A
$$

where $\sigma$ is the conductivity of the medium. Fock (3) defines the conductivity of the aperture as

$$
C=I / 2 \sigma B,
$$

where $B$ is the unknown constant appearing in the specifications (5.1), and our aim is to calculate this number, hence the conductivity $C$, in the limit when $\delta=1-(a / b)$ is small.

Integral equation formulation. The problem (5.4) is reduced to an integral equation, through the use of a Green's function $g\left(r, z ; r_{1}, z_{1}\right)$, described in the appendix, that is chosen to vanish when $r=b$ and to have zero normal derivative when $z=0$. It is found that $\psi\left(r_{1}, z_{1}\right)$ has the representation i.e.

$$
\psi\left(r_{1}, z_{1}\right)=\int_{a}^{b} g\left(r, 0 ; r_{1}, z_{1}\right) \psi_{z}(r, 0) r d r-\frac{1}{2} A b \int_{0}^{\infty} \frac{\partial g}{\partial r}\left(b, z ; r_{1}, z_{1}\right) b d z,
$$

$$
\psi\left(r_{1}, z_{1}\right)+\frac{1}{2} A r_{1}=\int_{a}^{b} g\left(r, 0 ; r_{1}, z_{1}\right) \psi_{z}(r, 0) r d r
$$

using equation ( $A 7)$ of the appendix. In particular, if $z_{1} \rightarrow 0$ and $a<r_{1}<b$, then (5.4) shows that $\psi$ assumes the value $-A b^{2} / 2 r_{1}$, and we are led to the integral equation

$$
-\frac{1}{2} A b\left(\frac{b}{r_{1}}-\frac{r_{1}}{b}\right)=\int_{a}^{b} g\left(r, 0 ; r_{1}, 0\right) \psi_{z}(r, 0) r d r, a<r_{1}<b,
$$

for the function $\psi_{z}(r, 0)$. 
It remains to express the constant $B$ in terms of the functicn $\psi_{z}(r, 0)$, and this is achieved as follows. Since $\phi \sim A z+B$ for large $z$, we have

$$
\begin{aligned}
B & =\int_{0}^{\infty}\left\{\phi_{z_{1}}\left(0, z_{1}\right)-A\right\} d z_{1} \\
& =-2 \int_{0}^{\infty}\left\{\psi_{r_{1}}\left(0, z_{1}\right)+\frac{1}{2} A\right\} d z_{1}, \text { from }(5.3), \\
& =-2 \int_{a}^{b} r \psi_{z}(r, 0) d r \int_{0}^{\infty} g_{r_{1}}\left(r, 0 ; 0, z_{1}\right) d z_{1}, \text { from }(5.7),
\end{aligned}
$$

i.e.

$$
B=\frac{1}{b} \int_{a}^{b}\left(\frac{b}{r}-\frac{r}{b}\right) r \psi_{z}(r, 0) d r
$$

using formula $(A 8)$ of the appendix. Thus if we define

$$
W_{1}(r)=\frac{1}{b B}\left(\frac{b}{r}-\frac{r}{b}\right) r \psi_{z}(r, 0),
$$

and

$$
K\left(r, r_{1}\right)=\left(\frac{b}{r}-\frac{r}{b}\right)^{-1}\left(\frac{b}{r_{1}}-\frac{r_{1}}{b}\right)^{-1} g\left(r, 0 ; r_{1}, 0\right),
$$

then equations (5.8) and (5.9) take the normalised form

$$
\int_{a}^{b} K\left(r, r_{1}\right) W_{1}(r) d r=-C / \pi b^{2}, \quad a<r_{1}<b,
$$

with

$$
\int_{a}^{b} W_{1}(r) d r=1
$$

The equations $(5.11,5.12)$, which together determine the potential field, and in particular the conductivity $C$, are of exactly the same type as have appeared in the earlier sections 2 and 3, and can be solved by the same procedure.

Approximate solution. An approximate solution for $W_{1}(r)$, and the constant $C$, is sought for small values of the parameter

$$
\delta=1-(a / b) .
$$

The variables $r, r_{1}$ and $W_{1}(r)$ are rescaled according to the transformations

$$
r=b(1-\delta x), \quad r_{1}=b\left(1-\delta x_{1}\right),
$$

and

$$
W(x)=b \delta W_{1}(r)=b \delta W_{1}(\dot{b}-b \delta x)
$$


The governing equations $(5.11,5.12)$ then become

$$
\begin{gathered}
\int_{0}^{1} K\left(x, x_{1}\right) W(x) d x=-C / \pi b^{2}, \quad 0<x_{1}<1, \\
\int_{0}^{1} W(x) d x=1,
\end{gathered}
$$

and are solved approximately by expanding the kernel $K\left(x, x_{1}\right)$ for small $\delta$. It is shown in the appendix that

$$
K\left(x, x_{1}\right) \sim K_{0}\left(x, x_{1}\right)=\frac{1}{4 \pi b \delta^{2}} \frac{1}{x x_{1}} \log \left|\frac{x-x_{1}}{x+x_{1}}\right|-\frac{3}{16 \pi b} \log \delta,
$$

whence a first approximation $W_{0}(x)$, and $C_{0}$, is defined by the integral equation

$$
\int_{0}^{1} K_{0}\left(x, x_{1}\right) W_{0}(x) d x=-C_{0} / \pi b^{2}, \quad 0<x_{1}<1
$$

i.e.

$-\frac{1}{\pi} \int_{0}^{1} \log \left|\frac{x-x_{1}}{x+x_{1}}\right| \frac{W_{0}(x)}{x} d x=x_{1}\left\{\frac{4 C_{0}}{\pi b} \delta^{2}-\frac{3}{4 \pi} \delta^{2} \log \delta\right\}, \quad 0<x_{1}<1$,

where use has been made of the scaling condition

$$
\int_{0}^{1} W_{0}(x) d x=1
$$

The integral equation (5.20) is a familiar one whose solution is known (cf. Cooke (2)). It can also be solved from first principles, since the approximate formula (5.19) corresponds to the two-dimensional problem of a steady flow past a plane with a strip of unit length protruding at right angles; this can easily be solved by conformal transformation and leads to the solution

$$
\frac{W_{0}(x)}{\dddot{x}}=\frac{x}{\left(1-x^{2}\right)^{\frac{1}{2}}}\left\{\frac{4 C_{0}}{\pi b} \delta^{2}-\frac{3}{4 \pi} \delta^{2} \log \delta\right\} .
$$

The constant $C_{0}$ is then found from condition (5.20), whence

and

$$
W_{0}(x)=\frac{4 x^{2}}{\pi\left(1-x^{2}\right)^{\frac{1}{2}}}
$$

$$
C_{0}=b\left(1+\frac{3}{16} \delta^{2} \log \delta\right) / \delta^{2} .
$$

To improve this estimate, the exact formula

$$
\begin{aligned}
C-C_{0} & =-\pi b^{2} \int_{0}^{1} \int_{0}^{1}\left(K-K_{0}\right) W\left(x_{1}\right) W_{0}(x) d x d x_{1} \\
& \sim-\pi b^{2} \int_{0}^{1} \int_{0}^{1}\left(K-K_{0}\right) W_{0}\left(x_{1}\right) W_{0}(x) d x d x_{1}
\end{aligned}
$$


is obtained from (5.16) and (5.19). An estimate for (5.25) requires a further expansion of the kernel $K\left(x, x_{1}\right)$, and is not pursued here since the next term, of order $\delta^{2}$, is found to have a coefficient that is expressed only in the form of a complicated definite integral involving modified Bessel functions.

Formula (5.23) complements the results of Fock (3) who deals with the regular limit of small $a / b$, and obtains a power series expansion for $C^{-1}$ up to terms of order $(a / b)^{12}$. The value obtained from (5.23) agrees well with Fock's prediction when $a / b=9 / 10$.

\section{Appendix. Properties of a Green's function}

The Green's function $g\left(r, z ; r_{1}, z_{1}\right)$ of section 5 is specified by

$$
\left(\frac{\partial^{2}}{\partial r^{2}}+\frac{1}{r} \frac{\partial}{\partial r}-\frac{1}{r^{2}}+\frac{\partial^{2}}{\partial z^{2}}\right) g=\frac{1}{r} \delta\left(r-r_{1}\right) \delta\left(z-z_{1}\right), \quad r_{1}<b, z_{1}>0,
$$

with

$$
g_{z}=0 \text { when } z=0, g=0 \text { when } r=b .
$$

Writing $g$ in the form of a Fourier integral,

$$
g\left(r, z ; r_{1}, z_{1}\right)=\frac{1}{2 \pi} \int_{-\infty}^{\infty} \hat{g}\left(r, r_{1} ; s\right)\left\{e^{-i s\left(z-z_{1}\right)}+e^{-i s\left(z+z_{1}\right)}\right\} d s,
$$

the transform function $\hat{g}$ is such that

$$
\left(\frac{\partial^{2}}{\partial r^{2}}+\frac{1}{r} \frac{\partial}{\partial r}-\frac{1}{r^{2}}-\delta^{2}\right) \hat{g}=\frac{1}{r} \delta\left(r-r_{1}\right)
$$

with $\hat{g}=0$ when $r=b$, and has the solution

$$
\hat{g}=-K_{1}\left(|s| r_{>}\right) I_{1}\left(|s| r_{<}\right)+\frac{K_{1}(b|s|)}{I_{1}(b|s|)} I_{1}(|s| r) I_{1}\left(|s| r_{1}\right)
$$

the functions $I_{1}$ and $K_{1}$ are modified Bessel functions. In particular then, when $z=z_{1}=0$, we have

$$
\begin{aligned}
g\left(r, 0 ; r_{1}, 0\right)=-\frac{2}{\pi b} \int_{0}^{\infty} K_{1}\left(\frac{t r_{>}}{b}\right) & I_{1}\left(\frac{t r_{<}}{b}\right) d t \\
& +\frac{2}{\pi b} \int_{0}^{\infty} \frac{K_{1}(t)}{I_{1}(t)} I_{1}\left(\frac{t r}{b}\right) I_{1}\left(\frac{t r_{1}}{b}\right) d t
\end{aligned}
$$

A useful related function is that obtained by integrating $g\left(r, z ; r_{1}, z_{1}\right)$ from $z=0$ to $z=\infty$, and is most easily calculated directly from equation (A1). Thus

$$
\left(\frac{\partial^{2}}{\partial r^{2}}+\frac{1}{r} \frac{\partial}{\partial r}-\frac{1}{r^{2}}\right) \int_{0}^{\infty} g d z=\frac{1}{r} \delta\left(r-r_{1}\right)
$$


with $\int_{0}^{\infty} g d z=0$ when $r=b$, and has the solution

$$
\int_{0}^{\infty} g\left(r, z ; r_{1}, z_{1}\right) d z=\int_{0}^{\infty} g\left(r, z_{1} ; r_{1}, z\right) d z=\frac{r r_{1}}{2 b^{2}}-\frac{r_{<}}{2 r_{>}} .
$$

In particular,

$$
\int_{0}^{\infty} \frac{\partial g}{\partial r}\left(b, z ; r_{1}, z_{1}\right) d z=r_{1} / b^{2}
$$

and

$$
\int_{0}^{\infty} \frac{\partial g}{\partial r_{1}}\left(r, z_{1} ; 0, z\right) d z=-\frac{1}{2 b}\left(\frac{b}{r}-\frac{r}{b}\right) .
$$

Asymptotics for small $\delta$. We require the asymptotic form of the function $g\left(r, 0 ; r_{1}, 0\right)$ when $r=b(1-\delta x)$ and $r_{1}=b\left(1-\delta x_{1}\right)$ with $\delta$ small.

The first integral of $(A 5)$ is readily dealt with, since it can be expressed (9) in the form of a hypergeometric function,

$$
-\frac{2}{\pi b} \int_{0}^{\infty} K_{1}\left(\frac{t r_{>}}{b}\right) I_{1}\left(\frac{t r_{<}}{b}\right) d t=-\frac{2}{b} F\left(\frac{1}{2}, \frac{1}{2} ; 1 ; r_{<}^{2} / r_{>}^{2}\right),
$$

whose known expansion (cf. (1)), with argument close to unity, leads to the result that

$$
\begin{aligned}
& -\frac{2}{\pi b} \int_{0}^{\infty} K_{1}\left(t-t \delta x_{>}\right) I\left(t-t \delta x_{<}\right) d t \\
& \quad \sim-\frac{1}{\pi b}\left\{-\log \delta-\log \frac{\left|x-x_{1}\right| e^{2}}{8}-\frac{1}{2} \delta\left(x+x_{1}\right) \log \delta\right. \\
& \left.\quad-\frac{1}{2} \delta\left(x+x_{1}\right) \log \frac{\left|x-x_{1}\right| e^{3}}{8}-\frac{1}{16} \delta^{2}\left(9 x^{2}-2 x x_{1}+9 x_{1}^{2}\right) \log \delta+O\left(\delta^{2}\right)\right\} .
\end{aligned}
$$

To deal with the remaining integral of $(A 5)$ we note that, for small $\delta$, the factor $I_{1}(t-t \delta x) I_{1}\left(t-t \delta x_{1}\right)$ appearing in the integrand is nearly the same as $I_{1}(t) I_{1}\left(t-t \delta\left(x+x_{1}\right)\right)$. Thus

$$
\frac{2}{\pi b} \int_{0}^{\infty} \frac{K_{1}(t)}{I_{1}(t)} I_{1}(t-t \delta x) I_{1}\left(t-t \delta x_{1}\right) d t=\frac{2}{\pi b} \int_{0}^{\infty} K_{1}(t) I_{1}\left(t-t \delta\left(x+x_{1}\right)\right) d t+R,
$$

where

$$
R=\frac{2}{\pi b} \int_{0}^{\infty} \frac{K_{1}(t)}{I_{1}(t)}\left\{I_{1}(t-t \delta x) I_{1}\left(t-t \delta x_{1}\right)-I_{1}(t) I_{1}\left(t-t \delta\left(x+x_{1}\right)\right)\right\} d t
$$

is small when $\delta$ is small. 
The first term of ( $A 11)$, which gives the main contribution when $\delta$ is small, can be written as a hypergeometric function, whence

$$
\begin{aligned}
& \frac{2}{\pi b} \int_{0}^{\infty} K_{1}(t) I_{1}\left(t-t \delta\left(x+x_{1}\right)\right) d t=\frac{2}{b} F\left(\frac{1}{2}, \frac{1}{2} ; 1 ;\left[1-\delta\left(x+x_{1}\right)\right]^{2}\right) \\
& \sim \frac{1}{\pi b}\left\{-\log \delta-\log \frac{\left(x+x_{1}\right) e^{2}}{8}-\frac{1}{2} \delta\left(x+x_{1}\right) \log \delta-\frac{1}{2} \delta\left(x+x_{1}\right) \log \frac{\left(x+x_{1}\right) e^{3}}{8}\right. \\
& \left.-\frac{9}{16} \delta^{2}\left(x+x_{1}\right)^{2} \log \delta+O\left(\delta^{2}\right)\right\} .
\end{aligned}
$$

It remains to deal with the correction term $R$ of formula $(A 11)$. This can be estimated by dividing the range of integration at $t=N$, where $N$ is a large number such that

$$
N \rightarrow \infty, N \delta \rightarrow 0 \text { as } \delta \rightarrow 0 .
$$

Over the interval $0<t<N$, we have $t \delta \ll 1$ and the modified Bessel functions may be replaced by their Taylor series approximations about $t$; for $t>N$, on the other hand, the Bessel functions have uniformly large arguments and may therefore be replaced by their appropriate asymptotic forms. This procedure leads to the estimate

$$
R \sim \frac{x x_{1}}{2 \pi b} \delta^{2} \log \delta+O\left(\delta^{2}\right)
$$

whence from $(A 10),(A 13)$ and $(A 15)$ we get

$$
\begin{aligned}
g\left(r, 0 ; r_{1}, 0\right) \sim \frac{1}{\pi b}\left\{\log \left|\frac{x-x_{1}}{x+x_{1}}\right|+\frac{1}{2} \delta\left(x+x_{1}\right) \log \left|\frac{x-x_{1}}{x+x_{1}}\right|\right. \\
\left.-\frac{3}{4} x x_{1} \delta^{2} \log \delta+O\left(\delta^{2}\right)\right\} .
\end{aligned}
$$

Finally, the kernel of equation (5.16) is

$$
K\left(x, x_{1}\right)=\frac{1}{4 \delta^{2} x x_{1}} \frac{(1-\delta x)\left(1-\delta x_{1}\right)}{\left(1-\frac{1}{2} \delta x\right)\left(1-\frac{1}{2} \delta x_{1}\right)} g
$$

and is found from $(A 16)$ to take the asymptotic form

$$
K\left(x, x_{1}\right) \sim \frac{1}{4 \pi b \delta^{2} x x_{1}}\left\{\log \left|\frac{x-x_{1}}{x+x_{1}}\right|-\frac{3}{4} x x_{1} \delta^{2} \log \delta+O\left(\delta^{2}\right)\right\} .
$$

\section{REFERENCES}

(1) M. Abramowitz and I. A. StegUn, Handbook of Mathematical Functions (Dover, 1965).

(2) J. C. COoKE, The solution of some integral equations and their connection with dual integral equations and series, Glasgow Math. J. 11 (1970), 9-20. 
(3) V. A. Fock, A theoretical investigation of the acoustical conductivity of a circular aperture in a wall put across a tube, C.R. (Doklady) Acad. Sci. URSS (N.S.) 31 (1941), 875-878.

(4) G. A. Grinberg and V. N. KURITSYN, Diffraction of a plane electromagnetic wave by an ideally conducting plane ring and the electrostatic problem for such a ring, Soviet Physics Tech. Phys. 6 (1962), 743-749.

(5a) G. R. KIRChHoff, Zur Theorie des Kondensators, Monatsber. König. Preuss. Akad. Wiss. Berlin (1877), 144-162. 1891).

(5b) G. R. KIRCHHOFf, Vorlesungen über mathematische Physik 3. bd (Leipzig,

(6) H. KoBER, Dictionary of Conformal Representations (Dover, 1952).

(7) F. G. LePPINGTON and H. LeVINE, On the capacity of the circular disc condenser at small separation, Proc. Cambridge Philos. Soc. 68 (1970), 235-254.

(8) F. G. LEPPINGTON and H. LEVINE, On the problem of closely separated circular discs at equal potential, Q.J. Mech. Appl. Math. 25 (1972), 225-245.

(9) I. M. Rhysik and I. S. GradsteIn, Tables of Series, Products and Integrals (Berlin, 1963).

(10) I. N. SNeddon, Mixed Boundary Value Problems in Potential Theory (NorthHolland, 1966).

(11) D. A. SPEnCE, Math. Res. Centre Tech. Report 1039 (University of Wisconsin, 1970).

(12) D. A. SPENCE, A Wiener-Hopf solution to the triple integral equations for the electrified disc in a coplanar gap, Proc. Cambridge Philos. Soc. 68 (1970), 529-545.

IMPERIAL COLLEGE, LONDON

Stanford University, California 